\title{
Low Dose-Rate High-Resolution Transmission Electron Microscopy of Group III - Nitride Electronic Device Structures Using a Direct Electron Detector
}

\author{
P. Specht ${ }^{1}$, R. Kirste ${ }^{2}$, Z. Sitar ${ }^{2}$ and C. Kisielowski ${ }^{3}$ \\ 1. University of California, Berkeley, Dept. of Materials Science \& Engineering, Berkeley, CA, USA. \\ 2. NC State University, Dept. of Materials Science \& Engineering, Raleigh, NC, USA. \\ 3. Lawrence Berkeley National Laboratory, Molecular Foundry, Berkeley, CA, USA.
}

In recent years direct electron detectors became available that dramatically enhance the quality of atomic resolution images because their Modulation Transfer Function (MTF) approaches unity in the low frequency range, single electrons can be detected well above noise levels, and the field of view is greatly expanded [1]. Thus, MTF corrections to recorded high resolution images become marginal and atomic resolution images of radiation sensitive materials can be recorded with dose rates below $1 \mathrm{e} / \AA^{2} \mathrm{~s}[2]$.

Emerging advantages for atomic resolution phase contrast imaging are discussed in the context of the development of native substrates for $\mathrm{AlN}$ and GaN that provided new opportunities for electronic device optimization. The epilayers in question contain multiple $\mathrm{Ga}_{\mathrm{x}} \mathrm{Al}_{1-\mathrm{x}} \mathrm{N}$ quantum wells with different $\mathrm{Ga}$ content, separated by AIN layers, and grown on an AIN buffer. The material is heavily strained due to the lattice mismatch between the various quantum wells. TEM samples were prepared via standard FIB liftout (cross-section), followed by a low voltage Ar ion mill (Nanomill, Fischione), to carefully thin and clean the FIB-induced damaged surface areas. Imaging is performed utilizing a Nelsonian illumination scheme in an aberration-corrected microscope with monochromated electron beam (TEAM, FEI). Typically, image series were recorded using the $\mathrm{K} 2$ camera [1] and reconstructed to obtain the electron exit wave function. It reveals the atomic structure of the semiconductor even if the dose rates are low and produces noise dominated single images (See Figure 1 A).

In a first set of experiments, we probed for an expectable dependence of the electron scattering process on dose rates and sample thickness [3], which can now be explored without modulating the results by the MTF. It is seen from the wedge-shaped sample of Figure 1 that dose rate variations yield very significant alterations of the dynamic scattering process, which can be observed by monitoring the forbidden (001) reflection of the hexagonal structure and its thickness dependence. Allowed reflections are modified within a factor of $\sim 2$ by dose rates. The experiments set the stage for an optimized recording regime of the entire structure (Figure 2) and it is possible to analyze all quantum well stacks and their respective strain fields including disturbances from threading dislocations. Results will be discussed in terms of improvements of electron microscopy and their impact on material sciences [4].

\section{References:}

[1] G McMullan et al., Ultramicroscopy 147 (2014), p. 156.

[2] D Zhang et al., Science 359 (2018), p. 675.

[3] C Kisielowski et al., Physical Review B 88 (2013), p. 024305.

[4] Work at the Molecular Foundry was supported by the U.S. Department of Energy under Contract No. DE-AC02-05CH11231. 

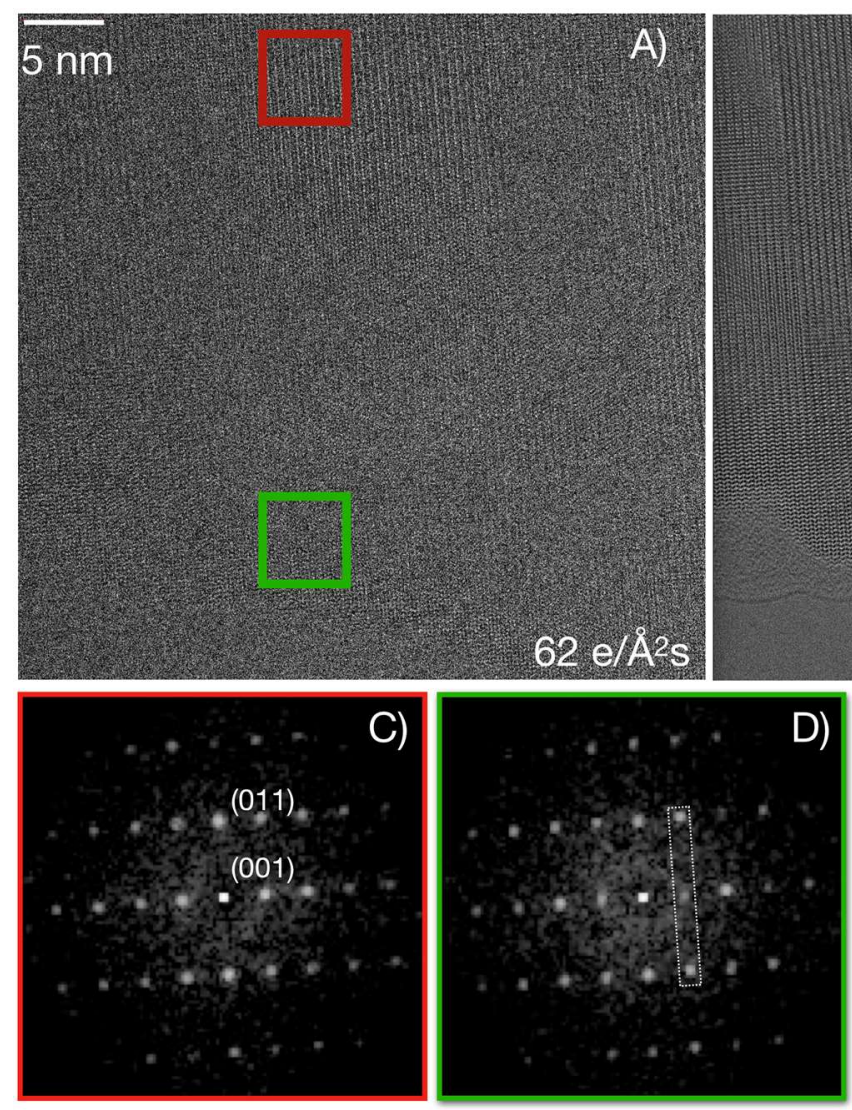

$250 \mathrm{~nm}$

$0.2 \mathrm{e} / \AA^{2} \mathrm{~s}$

0.2 e/A2s

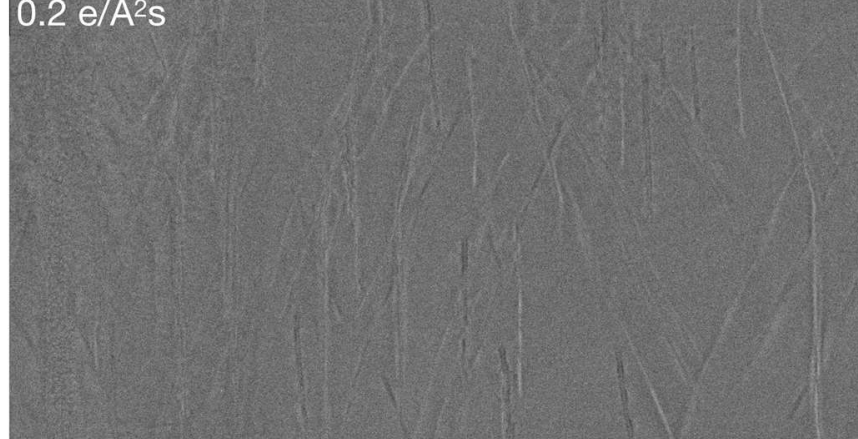

Stack

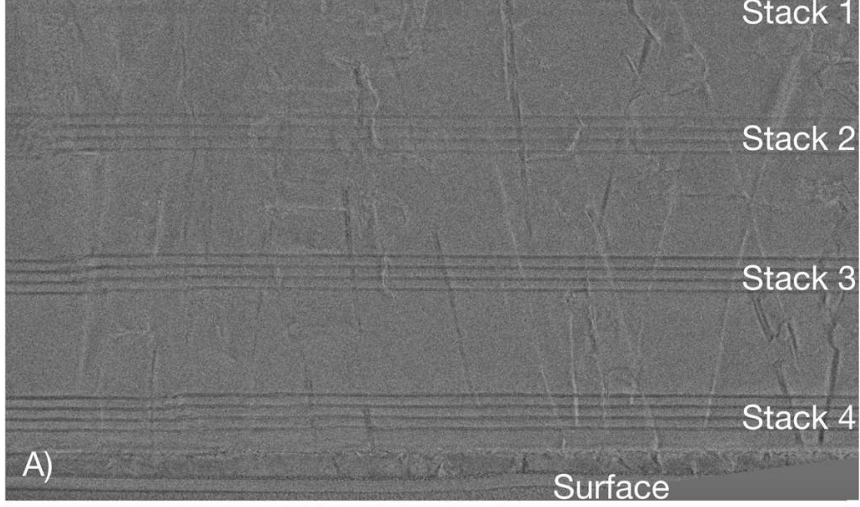

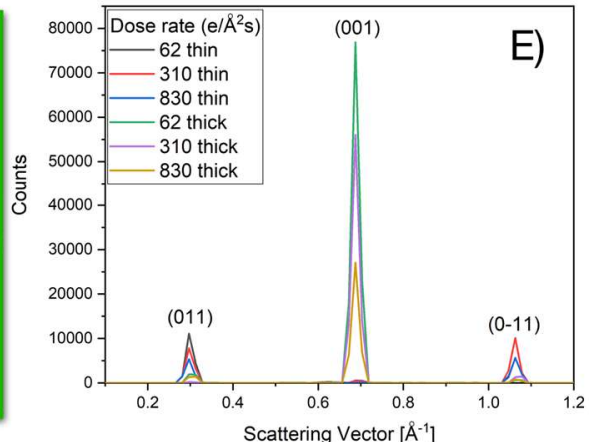

\section{$10 \mathrm{~nm}$}

B)

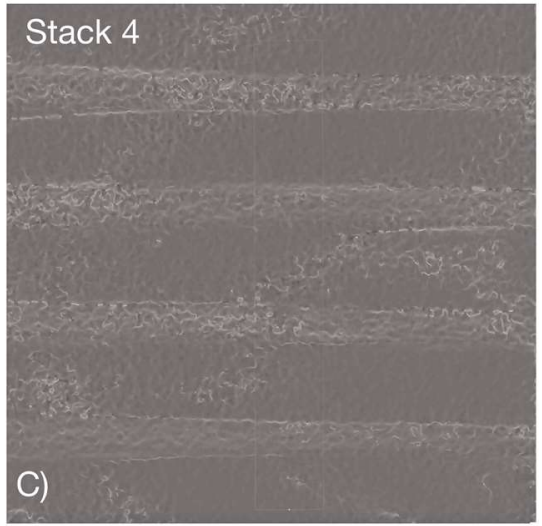

Figure 1.

A, B) Single images of $\mathrm{Al}_{\mathrm{x}} \mathrm{Ga}_{1-\mathrm{x}} \mathrm{N} / \mathrm{AlN}$ recorded with different dose rates. C, D)

Nano -

diffraction patterns of the reconstructed wave functions from thick and thin areas as indicated. (001) is a forbidden reflection. E) Dependence of the selected diffraction spots on thickness and dose rates.

Figure 2.

A) Large scale imaging of $\mathrm{Al}_{\mathrm{x}} \mathrm{Ga}_{1-\mathrm{x}} \mathrm{N} / \mathrm{AlN}$ quantum wells grown on sapphire. B) Magnified view of Stack 4. C) Principle strain map across the structure using a geometric phase analysis. 